\title{
Zusammenfassung der Ergebnisse
}

Richard Schaukals Wirken im schriftlichen Resonanzraum und seine Disposition verdeutlichen ein Bewusstsein für die Inszenierungsmöglichkeiten eigener und fremder Biographien. Dieses impliziert auch die Frage, bis zu welchem Punkt ein vermeintlich der Autonomieästhetik verpflichteter Dichter um 1900 Individualist bleiben konnte und ab wann er auf eine (soziale, berufliche, literarische, verlegerische) Gruppe zurückgreifen musste, um sein Schaffen in symbolisches und ökonomisches Kapital zu verwandeln. Schaukals Selbstbekenntnisse und privaten Briefe sind von diesem Thema ebenso durchzogen wie seine lebensgeschichtlichen Studien und fiktiven Werke, die mit auto-/biographischen Facetten und hybriden Autorkonzepten spielen.

$\mathrm{Zu}$ seinen biographischen wie dichterischen Selbstentwürfen zählen die Narrative Isolation und Subjektivismus; Schaukals genieästhetische Poetik verband sich mit dem Wunsch nach sozialer Distinktion und künstlerischer Unabhängigkeit. Diese Narrative und lebensgeschichtlichen Entwürfe standen mit seinem Schaffen als Kritiker und Dichter sowie mit seiner Rolle als Literaturvermittler und Agent der Kanonbildung in engem Zusammenhang. In Form von subjektivistischen Essays, die an eine seit dem Ende des 19. Jahrhunderts verbreitete Form der impressionistischen Literaturkritik anknüpften, propagierte und festigte Schaukal seine eigene Position im literarischen Feld, vermittelte zeitgenössische Dichter einem elitären Lesepublikum und wirkte an Kanonisierungsprozessen mit, die unter anderem darauf abzielten, Künstler wie E.T.A. Hoffmann in (neuromantische) Diskurse einzuschreiben. In späteren Jahren nutzte Schaukal seine biographischen Essays auch als antisemitisches Medium, Bücher wie Karl Kraus. Versuch eines geistigen Bildnisses (1933) sind Ideologieträger und entwerfen subjektivistisch ein Bild des Verfassers.

Biographien konstituieren sich zwangsläufig über Interaktionen: „[T]he biography is not one of a single individual but rather of an individual that unites all the characteristics of a group“. ${ }^{1}$ Auch wenn Schaukal Einsamkeit mit schöpferischem Potential gleichsetzte, ${ }^{2}$ standen seine Poetik und sein Imago als Dichter auf einem kollektiven Fundament; sein Schaffen war unmittelbar und zwangsläufig von Personen abhängig, von Vermittlern, Freunden, Gegnern und Lesern, auch wenn ihm dieser Gedanke missfiel, da er die Konstruktion

1 Giovanni Levi: The Uses of Biography. In: Theoretical Discussions of Biography, S. 89-111, hier S. 100.

2 Vgl. Schaukal: Beiträge zu einer Selbstdarstellung, S. 1.

Ә Open Access. (c) 2020 Cornelius Mitterer, publiziert von De Gruyter. (cc)BY Dieses Werk ist lizenziert unter der Creative Commons Attribution 4.0 Lizenz.

https://doi.org/10.1515/9783110619744-005 
einer auf sich selbst zurückgeworfenen Genialität ein Stück weit revidierte. Schaukal konzedierte zwar, dass es für seine „Bekenntnisangelegenheiten“ einer Adressatengruppe bedurfte, jedoch wären „Buch, Brief, Gespräch [...], Selbstgespräche vor Zuhörern [Dinge], auf die es mir nicht ankommt, die mir aber dazu als Mittel notwendig sind.“3

Sein Bekenntnisdrang verband sich mit dem Bewusstsein, Teil einer gesellschaftlichen Gruppierung zu sein, von der es sich schreibend ein Stück weit zu distanzieren galt. Bourdieu beschreibt dies mit Blick auf Flaubert folgendermaßen: „Schreiben setzt alle Determinierungen, alle grundlegenden Zwänge und Beschränkungen des gesellschaftlichen Daseins außer Kraft." ${ }^{4}$ Gesellschaftlich zu existieren bedeute, sozial markiert zu sein und von Gruppen abzuhängen, von ihnen gehalten zu werden und sich an sie zu halten, kurz: „,ihnen zuzugehören, in Netzwerke sozialer Beziehungen mit der Objektivität, Undurchdringlichkeit, Beständigkeit eines Dings eingebunden zu sein, die sich in Gestalt von Verbindlichkeiten, moralischen Schulden, Pflichten, also Kontrollen und Zwän-

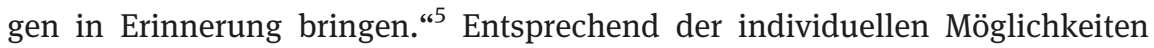
können diese Zwänge und sozialen Konventionen jedoch künstlerisch überwunden werden, so Bourdieu. ${ }^{6}$

Schaukals Werk ist nicht allein von konventionellen Narrativen über historische oder die eigene Größe geprägt, es zeigt auch eine bisweilen differenzierte Auffassung von Biographiewürdigkeit, auf die zum Beispiel seine Ein-Satz-Erzählung „Biographie“ aus dem Jahr 1902 anspielt, die den teleologischen Lebensverlauf eines liberalen Großbürgers karikiert. Zwar verehrte Schaukal sowohl prominente Historiographen des 19. Jahrhunderts (Carlyle, Mommsen, Treitschke und andere) als auch Protagonisten des literarischen Kanons (vor allem Goethe und Schiller). Aber noch viel stärker identifizierte er sich mit der Ästhetik und Biographie von Dichtern, die es um 1900 ein wenig wiederzuentdecken galt. Schaukals biographischer Auseinandersetzung mit E. T. A. Hoffmann, Heinrich von Kleist, Eduard Mörike oder Wilhelm Raabe (1831-1910) liegt die Geste des schöngeistigen Dilettantismus eines insgeheim doch intellektuellen Experten gesamteuropäischer Literaturgeschichte zugrunde. Wie die Dichter des Jungen Wien vertrat auch Schaukal die Auffassung, ,über nicht weniger als die gesamte abendländische Kultur seit der Antike verfügen zu können.“7

3 Schaukal: Beiträge zu einer Selbstdarstellung, S. 1.

4 Bourdieu: Die Regeln der Kunst, S. 58.

5 Bourdieu: Die Regeln der Kunst, S. 58-59.

6 Vgl. Bourdieu: Die Regeln der Kunst, S. 58-59.

7 Gotthart Wunberg: Einleitung. In: Die Wiener Moderne. Literatur, Kunst und Musik zwischen 1890 und 1910. Hg. von Gotthart Wunberg. Stuttgart 1981, S. 11-79, hier S. 61. 
Dabei versuchte er den Kanon auszuweiten, indem er zum Beispiel Romantik in einen erweiterten Klassikbegriff integrierte:

In der seit einigen Jahren bei uns um sich greifenden Begeisterung für die romantische Schule - kaum hat es je eine minder zutreffende Bezeichnung gegeben; eher könnte man von einer klassischen „Schule“ sprechen, die Romantiker waren lauter Einzelne, Individualisten - steckt ein gut Teil Snobismus. [.. .] Er ist also kein charakteristisches Merkmal jener Renaissance der Romantik. Aber man darf von seiner widerlichen Existenz nicht absehen. Es ist gewiß richtig: der banale Naturalismus - die Deutschen hatten, gründlich und grob, Ibsen mißverstanden - mußte eine Gegenbewegung hervorrufen. Aber die sogenannte Neuromantik war darum noch nicht Erlösung, weil sie einen Gegensatz vorstellte. Sie war etwas anderes, etwas vergleichsweise Neues und „zog“. Sie ist dahin [...], dahin wie der Rüpelreigen der „Konsequenten“ [Naturalisten, CM], Sie mußte an ihrer Blutleere umkommen. Die Treibhausblüte einer schwülen Nacht. Nichts mehr. ${ }^{8}$

Die Streichung des Präfixes ,Neu' und die Zusammenführung der Epochenbegriffe Romantik und Klassik hängt mit Schaukals gedehntem Generationenverständnis zusammen, dem nicht nur eine ästhetische, sondern auch eine zeitkritische, rückwärtsgewandte Note anhaftete. Indem er die Generationslagerung extrem ausweitete und sich in einen Zusammenhang mit Dichtern vergangener Jahrhunderte stellte, konstituierte er einen generationalen Zusammenhang, der den Generationenwechsel nicht nach biologisch messbaren Alterskriterien bestimmt, sondern nach geistig-ästhetischer Übereinstimmung. Schaukal fühlte sich etwa Novalis verbundener als den meisten seiner Zeitgenossen. ${ }^{9}$ Jene selbstbezogene Stilisierung der Vergangenheit, die Schaukal auch fotografisch inszenierte, wurde hier als nostalgischer Ausdruck und Anachronismus beschrieben. Die Portraitfotografien etwa waren beliebte Mittel, um Verbindlichkeiten herzustellen und dem Schreiben eine persönliche Note zu verleihen.

Die Analyse von Schaukals Korrespondenzen führte dabei zu dem Ergebnis, dass die Kanten seiner Netzwerke zwar sehr weitreichend, in aller Regel aber strukturell schwach waren. Vor allem die Verbindung zu Thomas Mann verdeutlicht eine Veränderung von zunächst symmetrisch verlaufenden Austauschprozessen (Informationen werden in gleicher Qualität gegeben und empfangen) zu asymmetrischen (einseitiger Informationsfluss).

Über den Kontakt zu Thomas und Heinrich Mann wollte sich Schaukal im literarischen Feld des deutschen Kaiserreichs etablieren. Dort waren die rechtlichen und ökonomischen Bedingungen für den Buch- und Zeitschriftenmarkt günstiger als in Österreich. Die Zusammenarbeit mit dem Illustrator und Maler

8 Schaukal: E.T.A. Hoffmann, S. 275.

9 Vgl. Schaukal: Frank Wedekind. Eine Porträtskizze. In: WE. Bd. 5, S. 66. 
Heinrich Vogeler, die die marktstrategische Hinwendung zum Buch- und Zeitschriftenschmuck um 1900 verdeutlicht, trug entscheidend zu Schaukals erster Etablierung im literarischen Feld Deutschlands bei. Der Leipziger Verleger Hermann Seemann publizierte 1902 das von Vogeler gestaltete Buch Pierrot und Colombine, davor hatten bereits die Verlage Tiefenbach (ebenfalls Leipzig), Pierson (Dresden und Leipzig) oder Schuster \& Löffler (Berlin) erste Werke des Dichters auf den Markt gebracht. Doch die Publikationen führten nicht zu einer dauerhaften Anbindung an die renommierten Kulturverlage der Zeit.

Für Schaukals Wirken als Schriftsteller ist die Verbindung nach München hervorzuheben, die ihm zwischen 1902 und 1910 eine günstige Position im Umfeld angesehener Zeitschriften einbrachte. Avalun, Die Gesellschaft, Die Insel, Jugend und Simplicissimus veröffentlichten seine Beiträge. In jene Jahre fällt auch der Kontakt mit einigen der aus heutiger Sicht bekanntesten Dichter, etwa mit Rilke, Thomas und Heinrich Mann sowie mit Hesse, die zum Teil für dieselben Zeitschriften tätig waren wie Schaukal.

Bis zu seinem Tod im Jahr 1917 publizierte der renommierte Verleger Georg Müller 22 Werke Richard Schaukals, ${ }^{10}$ darunter auch in sieben Auflagen Andreas von Balthesser. ${ }^{11}$ Der Kontakt zu Franz Blei, der als Insel-Redakteur dem München-Netzwerk angehörte, führte nach dem Ende der Zeitschrift zu zwei Veröffentlichungen beim Insel-Verlag. Schaukal generierte über den Redakteur soziales Kapital, das ökonomische und symbolische Gewinne nach sich zog. Blei vermittelte auch zwischen den Hoffmann-Verehrern Hans von Müller, Kubin und Schaukal; vor allem das Verhältnis zum Graphiker, Buchillustrator und Schriftsteller Kubin entwickelte sich zu einer lebenslangen Freundschaft und beruhte - im Gegensatz zu vielen anderen, intentional geknüpften Verbindungen - auf übereinstimmenden ästhetischen Vorlieben und weltanschaulichen Gemeinsamkeiten.

Auch wenn die Kontakte nach München Schaukals literarische Sichtbarkeit stärkten, gelang ihm nicht die gewünschte Vermittlung zu Eugen Diederichs, Samuel Fischer, Albert Langen oder - nach Anton Kippenbergs Übernahme zu Insel. Schaukals Einfluss beschränkte sich mehrheitlich auf Zeitungen und Zeitschriften, wodurch sich seine Präsenz auf einen schnelllebigen Teilbereich des literarischen Feldes erstreckte, der aufgrund wirtschaftlich-merkantiler, politischer und sozioökonomischer Faktoren im hohen Maß instabil war.

10 Vgl. die Liste der Schaukal-Veröffentlichungen im Anhang. Das einzige nach Georg Müllers Tod im Verlag publizierte Werk Schaukals war im Jahr 1931 der Band Gedanken.

11 Vgl. Schaukal: Beiträge zu einer Selbstdarstellung, S. 67. 
Während Hofmannsthal spätestens seit der Zusammenarbeit mit Richard Strauss seine literarischen Erzeugnisse ökonomisieren konnte und sich von der Lyrik abwandte, ${ }^{12}$ blieb Schaukal dieser Gattung sein Leben lang treu. Beiden gelang nicht der große Roman, auf den Hofmannsthal mit Andreas und Timon hinarbeitete. Thomas Mann hatte einen solchen mit Buddenbrooks vorgelegt, der seinen internationalen Ruhm begründete und ihm 1929 den Nobelpreis für Literatur einbrachte. Im Vergleich dazu verdankte etwa Hermann Bahr seinen Nachruhm nicht den literarischen Erzeugnissen, sondern der Fähigkeit zu antizipierenden Zeit- und Kulturanalysen sowie seiner Tätigkeit als Netzwerker und Kulturvermittler von internationalem Format.

Auf der Grundlage poetologischer Gemeinsamkeiten knüpfte Schaukal in der frühen Schaffensphase auch Kontakte zu Schriftstellern, die dem Prager Kreis zugeschrieben werden. Die Verehrung Liliencrons und das Interesse an der Neuromantik bildete die intersubjektive Basis dieses Austauschs. Vereinzelte Kontakte bestanden auch nach Berlin zum Sturm-Kreis und nach Wien zu Vertretern Jung-Wiens.

Die meisten Korrespondenzen Schaukals waren aber von geringer Dauer, wodurch sich kein dichtes Netzwerk etablieren konnte. Otto Julius Bierbaum wurde als relevanter Akteur für Schaukals Orientierung nach München ermittelt. Der Kontakt beschränkte sich jedoch auf eine kurze Zeitspanne, die Verbindung blieb uniplex. Nachdem Bierbaum die Insel verlassen hatte, setzte er sich bis zu seinem Tod im Jahr 1910 nicht weiter für Schaukal ein.

Von uniplexer Beschaffenheit waren auch die Verbindungen zu Brod, den Manns und zu Rilke; da im Gegensatz zu multiplexen Netzstrukturen die Bereitschaft zur Lösung von Konflikten nur gering ausgeprägt ist, brach Schaukals Kontakt zu diesen Akteuren ab, sobald ästhetische oder literaturbetriebliche Zwistigkeiten auftraten. Die Relationen entwickelten sich nicht zu freundschaftlichen Verbindungen mit starken Kanten, sondern blieben in aller Regel schwach geknüpfte, auf literarische Projekte oder ästhetische Themen begrenzte Austauschbeziehungen.

Auffällig ist des Weiteren, dass Schaukals Haltung im Laufe der Kontakte divergierte. Hermann Bahr und Arthur Schnitzler kontaktierte er in der klaren Absicht, literarischen Anschluss in Wien zu finden. Auftreten, Wortwahl und die mehr fordernd als bittend formulierten Vermittlungsgesuche führten aber zu einem Abstoßeffekt. Schaukal kann aufgrund seiner Poetik und Ästhetik

12 Vgl. Claudia Bamberg und Ilija Dürhammer: Dresden. Opernpremiere mit Ziergeflügel. In: Hofmannsthal. Orte. 20 biographische Erkundungen. Hg. von Wilhelm Hemecker und Konrad Heumann. Wien 2014, S. 250-268, hier S. 262-263. 
dem Dichterkreis des Jungen Wien zwar bedingt zugerechnet werden, über die geistige Verbindung hinauseichende, dauerhafte persönliche Kontakte kamen jedoch nicht zustande.

Entgegen Schaukals autonomieästhetischer Positionierung spielte ökonomischer Erfolg sehr wohl eine Rolle in den Briefwechseln, wie die Korrespondenz mit Thomas Mann oder Hermann Hesse zeigt. Doch an seinem eigenen Anspruch gemessen kann nicht von einem erfolgreichen Karriereverlauf als Schriftsteller die Rede sein. Dies ist mit Schaukals Ausscheiden aus den kulturellen Transferprozessen nach $1918 \mathrm{zu}$ begründen, die ihm vor dem Krieg als Vermittler (auch als Übersetzer) und Vermitteltem zugutekamen. Auffallend ist zudem, dass Schaukal gerade in der Hochphase seiner literarischen Betriebsamkeit nicht sonderlich oft (beruflich) reiste, wohingegen Hermann Bahrs literarisches Prestige auch auf seine internationale Vermittlertätigkeit und eine damit verbundene hohe Mobilität zurückzuführen ist. ${ }^{13}$

Schaukal versuchte in den ersten zwei Jahrzehnten seines beruflichen wie dichterischen Schaffens, Kunst und Geld miteinander zu vereinbaren. ${ }^{14}$ Sein Beamtenberuf, die vorteilhafte Hochzeit und eine Erbschaft machten ihn finanziell unabhängig, sodass er sich als Schriftsteller relativ frei entfalten konnte. Es ist kein Zufall, dass die frühen Jahre mit seiner kreativsten Schaffensphase und der intensivsten Netzwerktätigkeit zu maßgebenden Verlagen, Zeitschriften, Vereinigungen, Künstlern und Architekten der Moderne zusammenfallen. Bis etwa 1918 verbuchte er für alle Kapitalsorten Zuwächse, sozial und ökonomisch, kulturell wie symbolisch gewann er Einsatzmittel für seine Handlungen und Positionierungen in unterschiedlichen Feldern, nicht nur im literarischen. Das hängt vor allem mit seiner Tätigkeit als Autor für diverse Zeitungen und Zeitschriften zusammen. Ein zentraler Gedanke in Bourdieus Die Regeln der Kunst ist dem entsprechend, dass Künstler ihre Werke ab der Mitte des 19. Jahrhunderts zwei zentralen Vermittlungsinstanzen unterordnen mussten, da die ehemals im Kunstfeld herrschenden Fördergeber, also adelige und kirchliche Mäzene, weggefallen waren: Vor allem der Journalismus, aber auch Salons bestimmten zunehmend die neuen ,Regeln der Kunst" und beeinflussten die Vermittlung und Durchsetzung bestimmter Geschmacksvorstellungen. ${ }^{15}$ In Wien waren um 1900 außerdem Kaffeehäuser und Gaststätten Orte der ästhetischen Geschmacksbildung und -findung sowie der sozialen Vernetzung. Schaukal war in einigen wesentlichen, für die Etablierung im Feld der Literatur wichtigen Orten und Instanzen absent. Weder fand er

13 Vgl. Bachleitner: Eine soziologische Theorie des literarischen Transfers, S. 148.

14 Laut Bourdieu die „coincidentia oppositorum“; Bourdieu: Die Regeln der Kunst, S. 45.

15 Vgl. Bourdieu: Die Regeln der Kunst, S. 86. 
Anschluss an einen der kulturellen Salons in Wien, noch frequentierte er regelmäßig einschlägige Kaffeehäuser oder literarisch-kulturelle Zirkel, Gesellschaften und Vereinigungen.

Bourdieu begründet die von Manet ausgelöste ,symbolische Revolution“ mit dessen überaus günstiger Kapitalstruktur, die ihm einen großen Handlungsspielraum in den Feldern der sozialen Welt gestattete. Künstlerische Revolutionäre entstammen üblicherweise einem privilegierten, wohlhabenden Milieu; auf den ersten Blick stelle dies ein „soziales Paradoxon“ dar, so Bourdieu. Doch die Durchbrechung althergebrachter Wahrnehmungsmuster erfordere geradezu ein umfassendes Kapitalvolumen, das oft nur mit der affirmativen Verankerung in staatlichen Strukturen und besser gestellten Sozialschichten zu akquirieren sei. ${ }^{16}$

Hinzu kommt, dass Kreativität durch Wahrnehmungsveränderungen freigesetzt wird. Eine veränderte Perzeption ist in der Regel das Ergebnis von Kulturtransfers, ${ }^{17}$ in die auch Schaukal zur Zeit der Jahrhundertwende eingebunden war. Informationen, Symbole, Praktiken, auch Gegenstände zirkulieren in „multiplexen Verfahren“, wodurch sie ständig transformiert und re-interpretiert werden. ${ }^{18}$

Die multiplen Austauschbeziehungen führten bei Schaukal zwar zu keiner ,symbolischen Revolution', kennzeichnen ihn aber als Vertreter der Wiener Moderne, die in besonderer Weise durch Transferprozesse geprägt war. So wie das Junge Wien bewegte sich auch Schaukal in einem dynamischen Netzwerk künstlerischer Austauschbeziehungen, das nach Helga Mitterbauer aus vier Dimensionen besteht: (1) Kulturtransferprozesse in Netzwerken überbrücken räumliche Distanzen, werden (2) von Akteuren oder Gruppen getragen, verlaufen (3) über eine nicht linear oder chronologisch strukturierte Distanz und sind (4) von unterschiedlichen sozialen, kognitiven oder ideellen Konstellationen und wechselhaften Machtformationen geprägt. ${ }^{19}$

Auf Schaukal übertragen bedeutet dies, dass er die technisch-medialen wie infrastrukturellen Möglichkeiten nutzte, um sich mit künstlerischen Akteuren, Gruppen oder Institutionen in Frankreich, Italien, Deutschland, ÖsterreichUngarn, und vor allem dezidiert in Wien, auszutauschen (1 und 2). Die Transfers waren von unterschiedlicher Dauer beziehungsweise wurden zum Teil auch nach Jahren der Unterbrechung wieder aufgenommen (3). Kognitive Veränderungen spielten gerade für Schaukals Netzwerkagieren und seinen Austausch eine wesentliche Rolle. Literarische Bewunderung schlug in Distanzierung oder

16 Bourdieu: Manet, S. 24.

17 Vgl. Karl-Heinz Brodbeck: Entscheidung zur Kreativität. Darmstadt 1995.

18 Helga Mitterbauer: Dynamik - Netzwerk - Macht. Kulturelle Transfers „am besonderen Beispiel“ der Wiener Moderne. In: Ent-grenzte Räume, S. 109-130, hier S. 111.

19 Vgl. Mitterbauer: Dynamik - Netzwerk - Macht, S. 113. 
Verachtung um, wenn gleichrangige oder anfangs sogar weniger erfolgreiche Akteure ihn im literarischen Feld übertrafen. Vor allem sein Antisemitismus, verbale Ausfälle und die Politisierung beeinflussten zunehmend Schaukals Netzwerkstrategien und Handlungspläne (4).

Die veränderte politische Segmentierung der Gesellschaft ab 1919, in deren kulturpolitische Prozesse sich Schaukal einbrachte, hemmte seine dichterische Entfaltung. Seine Position in der sozialen Welt verschob sich von einer aktiven Partizipation am literarischen Feld hin zu einer immer passiveren Rolle in allen Feldern: auch in den Bereichen Politik und Bildung, die er für sich erobern wollte, blieb er nur mäßig erfolgreich. Der einstige Vertreter des ,L'art pour l'art' beteiligte sich schließlich sogar an der Auflösung der Autonomiebestrebungen im kulturellen Feld, weil er dieses für ideologische Zwecke zu nutzen begann (wie die ideologischen Anthologien der 1930er Jahre zeigen).

Schaukals Handlungen in den späteren Jahren sind mit Bourdieu als ,soziales Altern' zu bezeichnen. Damit ist nicht vordergründig der biologische Alterungsprozess gemeint, sondern die Anapassung der Wünsche und Erwartungen an die objektiv gegebene Lage. Die Dialektik zwischen Disposition und Position, also zwischen Angestrebtem und Erreichtem, manifestiert sich exemplarisch in Schaukals 1934 veröffentlichter Autobiographie, den Beiträgen zu einer Selbstdarstellung, die als Rechtfertigungsautobiographie im Zeichen sozialen Alterns gelesen werden kann:

\footnotetext{
Von dem, was ich in den letzten Jahren geschrieben habe, ist bis auf die „Gedanken“, die Ende 1930 herauskamen, noch nichts in Buchform geborgen. Die Ungunst der Verhältnisse, wohl auch die Mißgunst der dazu Berufenen hat's bisher verhindert. [...] Und meine schriftstellerische Tätigkeit hat sich unterm Druck der wirtschaftlichen Not nur vervielfacht. Die Ernte ist hoch gewachsen. Ich kann sie jederzeit verlassen. Sie wird nicht verloren gehen. ${ }^{20}$
}

Im Zitat wird ersichtlich, was ,soziales Altern“ bei Schaukal bedeutet: er findet sich rückblickend mit nicht erreichten Zielen und Positionen im Lebenslauf ab oder täuscht vor, die gegenwärtige Lebenssituation sei angemessen und gewollt. ${ }^{21}$

20 Schaukal: Beiträge zu einer Selbstdarstellung, S. 132.

21 Vgl. Bourdieu: Die feinen Unterschiede, S. 189. 
